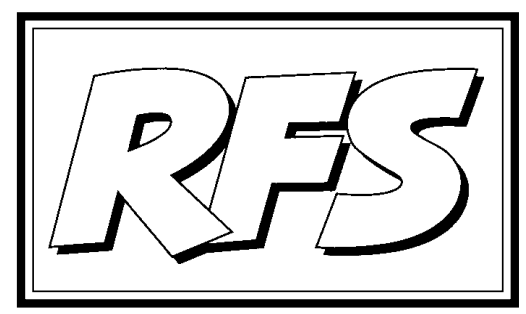

Revista de Fomento Social, 58 (2003), 539-558

\title{
Europa y la Crisis del Café. Un Plan de Acción
}

OXFAM INTERNACIONAL ${ }^{1}$

(Palabras clave: Crisis del café, Calidad del café, Barreras arancelarias. Key Words: Coffee Crisis, Quality of Coffee, Tariff Barriers)

'Cuando se afirma que gran parte de la pobreza mundial se concentra en las zonas rurales a la gente se le olvida aclarar que esto es debido especialmente a la volatilidad y a la caída a largo plazo de los precios de las materias primas...

'En la cuestión de las materias primas existe una especie de silencio conspirador. Las soluciones no son sencillas... Pero nada justifica la actual indiferencia.'

JacQues ChIRAC, Presidente de Francia, 21 de febrero de $2003^{2}$

1 Este trabajo fue escrito por Thomas Lines. Oxfam reconoce la ayuda de Leendert Sprey y Paul Goodison en su producción, y el autor quieretambién dar las gracias a Phil Evans, Phil Bloomer, Sophia Tickell, Marita Hutjes y J o Leadbeater por sus comentarios y consejo. Este es parte de una serie de estudios escritos para informar el debate público sobre temas de desarrollo y política humanitaria. Para más información, por favor, visite www.maketradefair.org.

2 Intervención de Jacques Chirac, Presidente de la República, en la 22a Conferencia de J efes de Estado de África y Francia, sobre el desarrollo agrario, p. 4. Texto publicado por el Gobierno Francés, traducido extraoficialmente por Oxfam. 


\section{La crisis del café}

La crisis actual del mercado del café deja a más de 25 millones de familias pobres enfrentando la ruina, incapaces de poner comida suficiente en la mesa, mandar a sus hijos a la escuela, o comprar las medicinas más básicas para cubrir las necesidades en materia de salud de sus familias. En regiones en las que se cultiva el café tan diversas como Etiopía, Nicaragua y Vietnam, las personas están al límite de la hambruna. Y las autoridades en Centroamérica muestran su preocupación ante los peligros de la inestabilidad social, económica y política que se deriva de la crisis ${ }^{3}$.

El comercio de café ha sido vital para el desarrollo.

Aproximadamente 70 países en desarrollo producen y exportan café, del cual, el 70 por ciento es cultivado por pequeños propietarios. Es, además la principal fuente de divisas para algunos de los países más pobres, proporcionando cerca del 80 por ciento de los ingresos por exportaciones en el caso de Burundi y el 70 por ciento en el de Etiopía.

Pero estos no son años buenos. El precio que reciben los productores pobres se ha derrumbado. El precio internacional de referencia, el café arábica que cotiza en la Bolsa de Nueva York, cayó por debajo de un dólar por libra sólo una vez durante los años ochenta, y por un breve periodo de tiempo; y tanto en 1977 como en 1997 subió por encima de los 3 dólares por libra durante el pánico de las heladas de Brasil. Pero en 2001 cayó hasta los 42 centavos por libra. En el último trimestre de 2002 subió a 70 centavos, aunque volvió a caer al final del año. Los precios en caída libre se traducen en que los países productores no pueden afrontar el gasto en salud y educación ni pagar la deuda externa, mientras los bancos nacionales van a la bancarrota.

La parte del precio final que reciben los cultivadores por los "granos verdes" no procesados ha caído en picado, del 64 por ciento del precio de venta al público en los EE.UU. en 1984 a sólo el 18 por ciento de ese precio en el $2001^{4}$. La investigación realizada por Oxfam en 2002 descubrió que los

3 Ver 'Central America moves to eliminate low-grade coffee,' (Centroamérica se moviliza para eliminar el café de baja calidad) declaración de las autoridades cafetaleras de Costa Rica, El Salvador, Hondura, Guatemala, Méjico y Colombia, 19 de Julio 2002 (www.ico.org/ed/ 19julio.htm).

4 Estos porcentajes ajustan el precio del grano verde por pérdida de peso para hacer que este precio sea comparable al precio minorista. Este tema, junto con el caso Ugandés, están tratados en detalle en Oxfam, (2002), pp. 20-25. 
cultivadores ugandeses de café sólo recibieron el 2,5 por ciento del precio de venta al público al que se vendió su café en el Reino Unido. Mientras, las compañías internacionales que controlan tanto el comercio como el tostado del café obtienen unos beneficios gigantescos. Se estima que el margen operativo de Nestlé en el café instantáneo se estima entre el 26 y el 30 por ciento del precio final al consumidor ${ }^{5}$. Esto refleja la concentración del mercado en manos de las grandes firmas tostadoras. Cualquier solución a la crisis del café pasa por incrementar el poder de negociación de los productores.

La crisis del café exige ahora una actuación internacional concertada. No obstante, los gobiernos de los países ricos han ignorado y se han desentendido de un sufrimiento que nunca habrían tolerado si hubiera afectado a sus propios ciudadanos. El Banco Mundial y la UNCTAD, las instituciones globales encargadas de hacer que los mercados de materias primas contribuyan al desarrollo, hasta el momento sólo han aplicado acciones preliminares.

Por ello, en septiembre de 2002 Oxfam y la Alianza Global para las Materias Primas y el Café (GLACC) propusieron conjuntamente un Plan de Rescate del Café, con una serie de medidas dirigidas a:

- Restablecer el equilibrio entre la oferta y la demanda.

- Recuperar la calidad y aumentar la productividad.

- Aumentar los precios y reactivar los medios de vida.

- Retener y construir capacidades con valor añadido.

- Establecer alternativas reales para el desarrollo rural.

Este estudio investiga cómo la UE puede ayudar en el logro de esas importantes metas.

\section{Soluciones convencionales de ayuda al café}

La Organización Internacional del Café (OIC) ya ha ofrecido algunas propuestas constructivas a la crisis del café y es imperante el apoyo de la UE a las mismas. Se basan en las siguientes medidas:

5 Oxfam, (2002), p. 26, notas de pie de página 43, 44. 
- un Programa de Mejora de la Calidad (PMC) para aumentar el mínimo de calidad de café que pueden exportar los miembros de la OIC (aprobado en la resolución 407 de la OIC);

- como medida a corto plazo, ayudar a los productores a eliminar la producción de café de baja calidad y no rentable, y ayudar a los agricultores a encontrar otros medios de vida a largo plazo.

El Director Ejecutivo de la OIC asistió en julio de 2002 a la destrucción de café costarricense de baja calidad y, en noviembre de 2002, al desvío de 63.000 sacos de café ( 60 kilos por saco) de café de baja calidad para usos distintos de la bebida. Estas medidas son parte del acuerdo regional de América Central para eliminar café de baja calidad, en apoyo al PMC.

Sin embargo, nadie dijo que la solución a la crisis del café fuera sencilla. Se necesita combinar elementos de diversificación económica, control de la producción, regulación de la calidad y otras medidas. Gran parte de la solución depende del compromiso de los propios países productores de café. El problema fundamental de la sobreproducción no se resolverá a menos que los productores encuentren vías para afrontarla de forma conjunta, con o sin ayuda de los países importadores. Muchos países productores están revisando sus acuerdos nacionales del sector y deberían, consecuentemente, eliminar to das las políticas que pudieran incentivar la sobreproducción o desincentivar la diversificación. Por ejemplo, debido a la importancia del café para la balanza de pagos, en al gunos países es ilegal arrancar cafetos (árbol del café). Sin embargo, arrancar estos cafetos puede estar justificado como un medio para reducir las pérdidas catastróficas de los agricultores.

Las principales líneas de ayuda a los países más pobres las determinan los Documentos de Estrategia de Lucha contra la Pobreza (PRSP en inglés) del Banco Mundial. Una crítica habitual a los PRSP es que no logran establecer los vínculos entre el comercio internacional y la pobreza doméstica. Este hecho está reconocido incluso oficialmente por la propia Comisión Europea ${ }^{6}$. Pero la crisis del café es una muestra evidente de la importancia de estos vínculos. Las soluciones a la crisis deben estar adaptadas a las

6 'En términos generales, sin embargo, la primera generación de PRSP ha fracasado a la hora de dar la importancia necesaria a los asuntos comerciales' (Comunicación de la Comisión al Consejo y al Parlamento Europeo sobre 'Comercio y Desarrollo: Ayudando a los Países en Desarrollo a Beneficiarse del Comercio', 19 de septiembre de 2002 [ref. no. COM (2002) 513 final], p. 20). 
necesidades y posibilidades locales -agrarias, económicas, climáticas, culturales o sociales-. Puesto que los PRSP se adaptan para cada país y se deben diseñar de forma participativa, deberían poder afrontar las necesidades específicas de los productores de café afectados en los países quecorresponda. El Fondo Común para los Productos Básicos también puede desempeñar un papel, al igual que los donantes bilaterales o multilaterales.

Por su parte, la UE ha utilizado recientemente medidas para enviar ayuda de forma urgente en otras áreas para proporcionar exactamente el tipo de ayuda que necesitan los productores de café de los países más pobres y sus familias. Los programas de emergencia habitualmente utilizados para los agricultores de la UE es el que necesitan urgentemente los productores de café, que son incluso más vulnerables. En Europa, se facilitan ayudas directas a los agricultores afectados por crisis específicas, desde los ganaderos británicos de las vacas locas hasta los productores de algodón griegos. Existen fondos de la UE disponibles inmediatamente que podrían financiar una situación como la del café. Un compromiso firme para acabar con esta crisis por parte de la UE supondría una clara señal de que está efectivamente preocupada por vincular comercio y reducción de la pobreza en el contexto de las conversaciones de la Ronda de Doha. Como un primer paso esencial, debería apoyar un diálogo que incluyese a la OIC y a los principales actores del mercado del café para discutir medidas orientadas a que el mercado del café beneficie a los más pobres.

La UE tiene el poder político, la habilidad técnica y el enfo que de desarrollo de ayuda-y- comercio necesarios en este contexto. De hecho, tiene más poder para influir en lo que ocurra en el mercado del café del quetiene ningún otro país o bloque comercial. Sus Estados Miembros son 15 de los 21 países importadores miembros de la OIC, y suman el 46 por ciento de las importaciones mundiales de café. (La participación de los EE.UU. en las importaciones es del 22.9 por ciento) ${ }^{7}$. La UE tiene acuerdos de cooperación con casi todos los países productores de café, 27 de los cuales son del grupo África, Caribe y Pacífico (ACP), y la mayoría de países restantes trabajan con la UE bajo el Programa de Asia y América Latina (ALA).

La UE ha comenzado a mostrar su compromiso al más alto nivel para acabar con la crisis del café. En noviembre de 2002 el Consejo de Asuntos

7 En 2000/01 los 15 estados miembros de la UE importaron 47.9m 60kg sacas de café, comparadas con los $23.8 \mathrm{~m}$ en EE.UU. de acuerdo con la OIC. 
Exteriores expresó su 'seria preocupación' por la situación de los países en desarrollo que dependían de las exportaciones de café, y afirmaba que la problemática 'necesitaba ser contemplada en el contexto más amplio del comercio y el desarrollo, desarrollo sostenible y lucha contra la pobreza'8.

\section{CUADRO 1 \\ Etiopía: una tragedia humana en la tierra de origen del café}

Etiopía es según algunos indicadores el país más pobre del mundo, con un ingreso nacional per cápita de sólo 100 dólares. También es el país de origen del café. Sin embargo, políticas internacionales perjudiciales, además de los bajos precios del café amenazan con convertir su principal exportación en una maldición casi tanto como en una bendición.

Tradicionalmente, las áreas de cultivo del café en el sur han estado entre las más prósperas de Etiopía: eran sitios a los que emigraba la gente cuando había hambruna en otras regiones. Pero ahora, mientras otra hambruna amenaza con enseñorearse, la gente en las regiones de cafetales como Kafa (de la que tomo su nombre el café) y Hararghe están entre los más afectados.

Según Mohammed Ali Indris, un agricultor de la provincia de Kafa, 'Hace cinco o siete años, yo producía siete sacos de grano rojo [caféno procesado] y eso era suficiente para comprar ropa, medicinas y servicios y para solucionar muchos problemas. Pero ahora, aún si vendo cuatro veces esto, no puedo cubrir todos mis gastos.

'Tres de los niños [de mi familia] no pueden ir a la escuela porque no puedo pagarles el uniforme' le dijo el Sr. Indris a Oxfam en Marzo del 2002.

El tamaño de la crisis económica de Etiopía quita el aliento. Entre 1997/98, un año fuerte para los precios del café, y 2000/01, el valor de las exportaciones de café de Etiopía cayó un 58 por ciento, de 420m de

8 'Comercio Internacional de Café', p. 18 de las Conclusiones de 2464a Reunión del Consejo de Asuntos Exteriores en Bruselas, 19 de noviembre de 2002. Disponible en: http://ue.eu.int/ Newsroom/makeFrame.asp?MAX $=1 \& B I D=71 \& D I D=73286 \&$ LANG=1\&File=/pressData/en/gena/ 73286.pdf\&Picture $=0$ en febrero de 2003. 
dólares de EE.UU. a 175m de dólares de EE.UU. Esto coincidió con un colapso del 48 por ciento en los términos de comercio con el resto del mundo. En el 2001, Etiopía tuvo que vender bienes por valor de cerca de $\$ 2$ para comprar el mismo valor que había comprado con $1 \$$ en años anteriores.

A pesar de esta dramática situación, la ayuda oficial de los países ricos para Etiopía cayó de \$883m en 1995 a \$693m en el 2000.

En el 2001, Etiopía también tuvo que pagar $\$ 41$ de deuda externa por cada $\$ 100$ de exportaciones. A Etiopía no se le ha perdonado hasta el momento ni un céntimo de los 5.6bn de dólares de deuda externa que tiene. Aún cuando en Diciembre del 2002 hubo una campaña internacional de protesta por la demanda de Nestlé de $6 \mathrm{~m}$ de dólares como compensación por la nacionalización de una compañía por parte de Etiopía en 1975. Para tener, por fin, opción a un perdón de la deuda, Etiopía tendrá que cumplir diez nuevas condiciones políticas, que son imposibles de lograr antes del último trimestre del 2003 como pronto - dos años más tarde de que se le prometiera el perdón-.

Recientemente, Pascal Lamy, Comisario de Comercio, afirmó ante el Parlamento Europeo que 'el hundimiento de los precios de café ha sido totalmente catastrófico'. Constituye un ejemplo 'que debería hacer reflexionar a aquellos que promueven la liberalización total de los mercados agrarios'. Conjuntamente con Poul Nielson, Comisario de Desarrollo, pretendía 'alcanzar una mejor coordinación de los distintos instrumentos de los que disponemos'9.

Para beneficiar a los países ACP, Oxfam Internacional solicita a la Comisión que se asegure que el superávit de fondos disponibles bajo el Fondo Europeo de Desarrollo (FED) se utilizan para reducir el sufrimiento humano derivado de la crisis del café y promuevan soluciones a largo plazo a la misma. Recientemente el comisario Nielson les dijo a los ministros africanos en una reunión en Burkina Faso,

9 Respuesta de Pascal Lamy en representación de la Comisión Europea a la pregunta no 41 del parlamentario de los Verdes, Camilo Nogueira Román (order paper item H-0008/03), en la sesión del Parlamento Europeo en Estrasburgo. Las citas han sido traducidas extraoficialmente por Oxfam. 


\begin{abstract}
'Al final de este año [2002] habremos acumulado alrededor de 11 billones de euros en dinero no desembolsado, comprometido o no comprometido, de los 60 , 70 y 8 ㅇ FED... Nuestra propuesta es que examinemos, junto con todos y cada uno de los socios de ACP, las posibilidades de liberar y atribuir viejos fondos al tiempo que utilizamos la nueva flexibilidad en toda su extensión en el 9o FED. Déjenme - para evitar cualquier malentendido- resaltar que la idea no es rehacer las estrategias de los países, sobre las que acabamos de ponernos de acuerdo, sino acelerar la entrega y abrir la posibilidad a iniciativas más amplias' 10 .
\end{abstract}

En consonancia, Oxfam Internacional insta a la Dirección General de Desarrollo a que trabaje con otras áreas de la Comisión en un programa de ayuda para los productores de café en los países más pobres. También solicitamos a la Comisión y a los Estados Miembros que favorezcan que los principales programas de desarrollo, especialmente el mecanismo de los PRSP/Países Pobres Altamente Endeudados, incluyan algunas medidas para ayudar a los productores de café a que continúen su producción de forma rentable 0 , cuando sea necesario, a que encuentren alternativas al café.

Oxfam Internacional propone las siguientes medidas para su valoración.

1. Incremento del volumen dela a yuda para ayudar a los productores y trabajadores del café. Referido a los países ACP, y que debería ser financiado con fondos reasignados del FED. Ayudaría a los agricultores a encontrar una vía a través de los problemas que impone la crisis y que son más difíciles de tratar. Se daría prioridad a los países productores de cafémás pobres y dentro de éstos a los pequeños cultivadores más pobres. Establecido mediante consultas con la OIC, el Programa debería asistir en estas áreas:

- Diversificación rural. La asistencia de la UE debería ayudar a los productores a abandonar la producción de café de baja calidad y del café no rentable, y a diversificar mediante otras cosechas o actividades no agrícolas. Debe ser proactiva y estratégica, ayudando a los productores y países que ya están haciendo los cambios necesarios y apoyando a los más necesitados.

La diversificación es una cuestión compleja, dado que la situación de cada agricultor es única, y sólo puede funcionar si los propios agricultores toman las principales decisiones y si las implicaciones en términos de recursos son realistas. Habrá mayor seguridad de éxito si se hace en alianza

10 Declaración de apertura del Comisario Poul Nielson con ocasión de la 2a Conferencia Ministerial África-Europa, Ouagadougou, 28 de Noviembre 2002. 
con programas nacionales, más amplios, de diversificación agrícola. En algunos lugares puede requerir una revisión de las prácticas actuales. Por ejemplo, en el norte de Malawi el FED está actualmente ayudando a la rehabilitación de la producción de café, con el objetivo de potenciar los ingresos en divisas ${ }^{11}$. En otros lugares cualquier reducción en la extensión de la tierra utilizada para cultivar café puede reducir el acceso de los agricultores al crédito o tener consecuencias adversas en el medio ambiente. La ayuda tendrá que superar todos esos efectos en cadena derivados de la reducción de la sobreproducción.

- Acciones que refuercen el poder de negociación de los productores de café. Esto se puede lograr por diversos medios, uno de los cuales es la aportación de información pertinente sobre el mercado. Los pequeños productores en las zonas altas de los países pobres no están bien situados para saber lo que pasa en los mercados internacionales. Esto los coloca en una posición de debilidad frente a los intermediarios que sí tienen esa información; puederesultar difícil cuestionar una oferta de precio y negociar. Esos agricultores están en la clásica posición de quienes tienen que aceptar un precio. Se deben encontrar formas para proporcionarles esta información en el futuro.

Los pequeños productores pueden también conseguir mejores acuerdos si negocian con compradores y proveedores colectivamente, como miembros de una cooperativa de producción o comercialización. Las cooperativas pueden atraer crédito con más facilidad y comprar los insumos esenciales, así como abrir camino hacia áreas del mercado de alta calidad. La UE debe apoyar la creación y desarrollo de cooperativas de productores de café.

Los servicios de extensión proporcionan a los agricultores información técnica acerca de cómo cultivar y cómo cosechar, además de asesoría sobre oportunidades de mercado. Actualmente, en los países productores de café este servicio se presta de forma irregular, pero en un momento en el cual el mercado del café se está haciendo cada vez más diferenciado, con más oportunidades de añadir valor en sectores especializados, tal asesoría tendrá más importancia que nunca. Debe ser apoyada.

Finalmente, el acceso a créditos e insumos puede mejorar mucho la posición de los productores casi en cualquier mercado. El crédito durante la

11 Sprey, L., (2002), p. 7, citando un estudio hecho en 2000 para el desarrollo de cultivadores de café pequeños propietarios en el Norte de Malawi. 
estación del crecimiento les permite pagar los insumos necesarios (tanto la mano de obra como los insumos físicos), y poder elegir en alguna medida el momento de vender la cosecha. Sin crédito, los productores son presa fácil de los comerciantes. Con frecuencia el apoyo más efectivo a los productores se encuentra en esta área.

2. Un plan de calidad extra. Tanto el Consejo como la Comisión han expresado su apoyo al Programa de M ejora de la Calidad de la OIC. Hay varias vías prácticas para hacerlo. El mercado del café se esta diferenciando cada vez más, con precios altos para ciertos productos "nicho"12. Esto refleja desarrollos similares en los mercados del vino y el whisky en décadas anteriores. Se paga extra por tres clases de café (que no se excluyen mutuamente): beneficioso para el medio ambiente (orgánico o a la sombra), el de comercio justo, y el 'gourmet' o café de 'especialidad' (de un país en particular o una sola finca).

Los productores de café necesitan contactos en los mercados de los países ricosy, con frecuencia, financiación exterior, antes de poder desarrollar tales líneas. Dentro de las estructuras del mercado se han desarrollado ya nichos orgánicos y de comercio justo. Ambos se benefician de la certificación independiente (ejemplo: la Asociación de Suelos del Reino Unido marca la producción orgánica, y la Fundación del Comercio Justo lo hace con los bienes comercializados de forma justa). Si hay un papel en esta área para la asistencia de los donantes, consistiría solamente en poner a los productores y a los compradores en contacto, apoyando los principios establecidos por la Asociación Europea de Comercio Justo y el movimiento de agricultura orgánica.

Algunos cafés de origen se están diferenciando del grueso del comercio "masificado", algo similar a las denominaciones de origen controladas para vinos y whiskys de una sola malta. A partir de pequeños volúmenes, estos últimos han obtenido poco a poco grandes porciones de sus respectivos mercados internacionales. Los cafés de alta calidad de un solo país siempre han conseguido precios más altos, pero hay signos de que esta categoría se está institucionalizando más. Esto es una ventaja evidente para los productores, siempre que logren obtener una parte justa de los márgenes ofertados.

12 Esto está bien analizado en Fitter And Kaplinsky, (2001). 
La UE tiene una rica experiencia en campos similares que puede ser de ayuda en este proceso. Debe investigar si un sistema formal de certificación sería beneficioso (como para los productos orgánicos y el vino con denominación de origen), o si la diferenciación debe continuar limitándose a la marca de comercialización (como en los whiskys de una sola malta). Una ventaja de la certificación es que pueden imponer requisitos sobre temas como las prácticas laborales, que un proceso sólo dirigido al mercado ignoraría. Por otra parte, no es deseable una proliferación de planes de certificación.

3. Destrucción de stock. Los beneficios del Programa de Mejora de la Calidad de la OIC llegarán demasiado tarde para aliviar la crisis humanitaria a no ser que, como una medida excepcional, se destruya una modesta cantidad de los stocks de peor calidad. La OIC estima que la destrucción de 5 millones de sacos, a un coste de 100 millones de euros, podría traducirse en una subida de precios del 20 por ciento respecto al precio promedio del 2000/01 -proporcionando entre 700 y 800 millones de euros en ingresos adicionales por exportación a los países productores de café-. Esto produciría un alivio inmediato al sufrimiento, estimularía la actividad de los gobiernos productores y proporcionaría el tiempo necesario para establecer soluciones a largo plazo. LA UE y otros donantes deberían proporcionar financiación sustancial para la destrucción puntual del café de peor calidad almacenado en los países importadores.

Y la necesidad de tal apoyo es ahora mayor que nunca. No ha habido nunca antes un hundimiento tan dramático en el mercado del café, y eso hace aún más urgente la necesidad de utilizar instrumentos de desarrollo. El mercado podría encontrar su propia solución forzando a los cultivadores a abandonar la producción. Ya está pasando eso en muchos casos. Pero sin intervención de los donantes o de los gobiernos, serán aquellos cultivadores que ya son los más pobres los que más sufran. Aquellos que tengan recursos para invertir o los que ya producen café de alta calidad pueden mejorar aún más su posición. El problema de aquellos cuyo café es de baja calidad es más complicado. Tienen que encontrar medios de vida alternativos, pero la experiencia demuestra que los agricultores más pobres son generalmente los menos capaces de asumir el riesgo del cambio o de hacer la inversión necesaria. 
Los mercados de materias primas en general, no sólo el del café, están, en este momento, enfrentándose a una crisis. Son necesarios abundantes recursos por parte de los países ricos. A largo plazo, será importante revisar qué ha funcionado y qué no ha funcionado en la puesta en práctica de programas de este tipo, incluyendo el establecimiento de sistemas de entrega para un rápido desembolso. Esto puede ser utilizado como base para una respuesta más elaborada a las necesidades de los países en desarrollo dependientes de materias primas, al estilo de la Comunicación sobre Materias Primas a la que ya se ha comprometido la Comisión.

\section{Compensación financiera}

Bajo el Acuerdo de Lomé, la UE aplicó el programa Stabex para compensar a los países ACP que sufrían pérdidas de sus ganancias por exportaciones debido a los movimientos de los mercados de materias primas. El presidente de Francia Chirac ha lamentado públicamente que ya no se haga uso del Stabex y sugirió incluso que los principales países industrializados deberían de importar su café y otras materias primas a precio garantizado ${ }^{13}$.

El Stabex proporcionaba a los países beneficiarios la liquidez que tanto necesitaban, especialmente en los primeros años, los desembolsos eran rápidos, automáticos y predecibles. El fondo Stabex hubiera sido útil como respuesta al colapso de los precios del café y un buen sustituto; ahora es completamente necesario un sistema equivalente. Durante las crisis previas de café, se destinaron 978 millones de Ecus para cubrir las pérdidas de 17 países ACP dependientes del café en el período 1990-94. A pesar de que hubo ciertas quejas porque los desembolsos se hicieron más lentamente que años anteriores, en la evaluación del Stabex de 1998 se elogió estos pagos por su papel en la reestructuración del sector del café.

En comparación, no se ofrecieron más que 642 millones de euros a ocho

13 'El algodón, el café y el cacao aseguran los ingresos y la supervivencia de millones de productores y trabajadores agrarios, de los más pobres del mundo y más desprotegidos... Por lo tanto, yo propondré a los demás miembros del G8 e instituciones internacionales que trabajan en este campo para reabrir el tema. Muchas vías merecen ser exploradas... [Una de ellas es] adaptarse al tratamiento de la deuda de los países pobres en función de la evolución de los precios de materias primas... Quizás nos equivocamos al abandonar los mecanismos del STABEX, incluso si no funcionó perfectamente. Podríamos pensar en vías de importar parte de su producción [africana] a precios garantizados.' Intervención de Jacques Chirac, op. cit., p. 4. 
países exportadores con el equivalente "B" en el noveno FDE. Los procedimientos para utilizar este dinero son más complejos, y hasta que los países Miembros de la UE no ratifiquen el Acuerdo de Cotonou, estos fondos no se pueden desembolsar legalmente. Por lo tanto, la rápida aplicación resulta menos probable. Teniendo en cuenta las crecientes necesidades de los países ACP más pobres por culpa de la crisis del café se podrían utilizar los fondos de ayuda de la UE. De esta manera se beneficiaría a la población rural sin que ello significase hacer ninguna referencia a los compromisos incluidos en las estrategias país de la UE. Una décima parte de los fondos disponibles del FDE (1.1. billones de euros de los 11 billones) es dinero del programa Stabex que aún no se ha gastado. Con vistas a la severa crisis actual, Oxfam pide a la UE que ofrezca 750 millones de euros de los fondos disponibles del Stabex a todos los países ACP afectados por la crisis del café, en proporción a sus necesidades.

El requisito más importante es el rápido desembolso que se tiene que dar para que los productores puedan tener un rápido alivio a la crisis. La ayuda debería centrarse en la balanza de pagos y en las ayudas presupuestarias, cuanto más descentralizada sea la ayuda mejor. Aquellos países que tengan un sistema administrativo apropiado, esto podría significar ayudar a las autoridades locales o a los comités de desarrollo. Un ejemplo exitoso de este tipo fue la ayuda que la UE proporcionó a cada comunidad local en Ruanda, quien otorgó alrededor de 1 euro por persona lo que significó una importante inyección de flujos en la economía. Los comités locales de desarrollo fueron los que decidieron cómo hacer uso de ese dinero.

En las zonas donde se cultiva el café en Uganda la situación es tan extrema que la gente no puede permitirse pagar el impuesto de 2 euros anuales por persona. Esto ha significado una importante caída de los presupuestos que afecta directamente a los servicios esenciales. Esta brecha podría ser rápidamente eliminada utilizando el dinero que no se ha gastado del Stabex de la manera que ya se ha propuesto. Las necesidades en las zonas donde se cultiva el café en Etiopía son aún más acuciantes teniendo en cuenta que ya hay escasez de alimentos (ver Cuadro 1). Muchas de las necesidades inmediatas de los productores de café son locales y podrían ser solventadas de esta manera. 


\section{CUADRO 2 \\ Las desgracias ocultas del café en África}

Las variedades de café, arábica y robusta, vienen de África y más de 5 millones de productores lo cultivan en más de la mitad de los países en el continente. Pero últimamente la producción se ha estancado, su cuota de mercado mundial ha caído y apenas se escucha su voz en el mercado del café. No se le ha dado la importancia necesaria a la gravedad de la crisis del café en África, porque la producción de cada uno de los países es generalmente pequeña como porcentaje de la producción mundial, a pesar de que puede ser muy importante para la economía nacional. Por ejemplo, en 2001, Burundi sólo exportaba el 0,3 por ciento del total mundial, pero suponía en 50 por ciento de sus ingresos de divisas. Una reducción del 50 por ciento en el precio del café significó una reducción del 79,6 por ciento de la cuota de las exportaciones respecto a 3 años antes.

En algunos países, habitualmente como resultado de una guerra, se ha hundido la producción de café. Antes de su independencia, Angola era el cuarto mayor productor del mundo, alcanzando un máximo de 4 millones de sacos al año, pero en 2002, exportó sólo 9.300 sacos. El volumen de exportaciones de la República Democrática del Congo cayó un 90 por ciento entre 1996 y 2000, y la contribución del café a los ingresos por exportaciones pasó del 15,9 al 1,6 por ciento.

En total, la cuota de mercado mundial de África ha pasado del 30 al 15 por ciento en los últimos 30 años, mientras que los ingresos derivados del café han caído un 65 por ciento en seis años. Esto ha supuesto una enorme pérdida de influencia política del continente en el mundo del café. Esto ha aumentado la crisis económica en muchos países, lo que significa que algunos no pueden permitirse enviar delegados regularmente a la OIC, y el predominio del café Robusta, de menor calidad, en su producción (con ciertas excepciones notables como Etiopía y Kenia).

En octubre de 2002, la Organización Interafricana del Café aprobó la Declaración de Malabo. Ésta solicitaba a sus miembros que adoptaran medidas de diversificación para aumentar el beneficio de los productores de café, promover el consumo doméstico y apoyar las resoluciones 407 sobre la calidad del café y 413 sobre la entrada de Estados Unidos en la OIC. 


\section{Prácticas comerciales}

El comercio puede jugar un papel más importante que la ayuda en la reducción de la pobreza, y la UE puede lograr muchas cosas mediante las políticas y los procedimientos del comercio internacional. El mercado del café es muy inhabitual entre los mercados agrícolas globales por estar casi totalmente liberalizado. En 1990 los antiguos mecanismos de regulación de los precios y las exportaciones del Acuerdo Internacional del Café estaban ya eliminados y muchos países productores habían sido persuadidos de no intervenir en él por medio de consejos de comercialización estatales u otros mecanismos. El problema del mercado del café es en realidad el del fracaso de la liberalización, no el de su insuficiencia.

Oxfam se felicita de que la Comisión haya prometido tomar medidas en el ámbito de los mecanismos comerciales. Por otra parte, la Comisión se ha comprometido a llevar a cabo un control de la calidad del café relativa a la Resolución 407 de la OIC "en colaboración estrecha con el sector privado". Cada trimestre mandará a la OIC "Ia información recibida de la Federación europea del café sobre el cumplimiento de los requisitos de calidad por parte de los exportadores de café"14.

Este es un paso importante pero insuficiente. La experiencia en campos similares lleva a Oxfam a pedir a la Comisión que afiance la credibilidad de estas medidas añadiéndoles el control independiente del café que entra en los mercados de la UE, y la publicación rápida de los resultados. Esto aportará la transparencia necesaria acerca de qué países y compañías son los que comercian por debajo de los estándares de la OIC. La forma más fácil de conseguirlo es que las autoridades aduaneras de los Estados Miembros comprueben la calidad del café importado, la contrasten con el certificado obligatorio de calidad de la OIC, y hagan públicos y accesibles los resultados tanto para los exportadores como para los importadores. El coste para la UE sería insignificante. El apoyo al esquema de la OIC y, en definitiva a los productores de café más pobres, sería notable. Actualmente todos los costes derivados de la aplicación del esquema de la OIC recaen en los países productores, a pesar de las restricciones financieras que sufren como consecuencia de la propia crisis del café.

14 Respuesta escrita fechada el 18 de Diciembre 2002 de la Comisión Europea a la pregunta oral del europarlamentario Nicholas Clegg no. H- 0748/02 sobre 'El Plan de la Organización Internacional del Café para la Mejora de la Calidad del Café'. 
Quedan dos vías por las cuales las barreras de la UE al comercio todavía impiden una recuperación en la crisis del café. Las propuestas para revisar las reglas internacionales para ambas vías fueron recientemente presentadas como parte de las negociaciones de la Ronda de Doha en Ia OMC14 ${ }^{15}$. Los negociadores de la Comisión deberían de presionar para que se alcanzase un resultado favorable en el Acuerdo sobre Agricultura de la OMC en ambas áreas.

En primer lugar, los aranceles a la importación de entre 7,5 y 9 por ciento sobre el café procesado más allá de la fase de grano verde, de ciertos países que no son ACP. Esto impide efectivamente a esos países aumentar su participación en el valor añadido asumiendo progresivamentedistintas fases del procesamiento -una estrategia básica de desarrollo-. El "Primer Borrador de Modalidades" de las negociaciones de agricultura de la OMC, publicado en febrero de 2003, proponía que los países desarrollados deberían garantizar "la liberalización de la mayor parte de los productos tropicales, tanto para los primarios como para los procesados"16. Oxfam solicita a la UE que este texto se convierta en requisito obligatorio, y pide a la Comisión que actúe de forma inmediata para eliminar los aranceles sobre el café procesado, adelantando el acuerdo final del texto de Doha.

En segundo lugar, muchas alternativas potenciales a la producción del café se pueden dar por descartadas debido a que la Organización Mundial del Comercio permite a los países ricos volcar productos agrícolas subsidiados en condiciones de dumping en los mercados de los países en desarrollo y, de esa forma, restringir los mercados de cultivos a los que los agricultores podrían acceder para diversificar. Uganda pretende diversificar su producción más allá del café pero sus intentos se ven frustrados por la prohibición de las exportaciones pesqueras por razones fitosanitarias y también por la falta de acceso a los mercados del maíz y de azúcar ya que no pueden competir con las exportaciones subsidiadas de la UE ${ }^{17}$.

Durante la actual Ronda de Doha de conversaciones sobre comercio mundial la UE aceptó retirar todos los subsidios a la exportación, tanto directos como indirectos. Se debería asegurar que se va a incluir en la

15 'Negociaciones de Agricultura: Primer Borrador de Modalidades para Mayores Compromisos', documento de la OMC preparado por Stuart Harbinson, 12 de febrero de 2003.

16 Id. párrafo 9.

17 Según la declaración de Louis Balinda, Representante de Uganda ante la UE, en la Mesa Redonda de Oxfam sobre la Crisis Global del Café, Bruselas, 8 de enero de 2003. 
revisión del Acuerdo sobre Agricultura un mejor acceso a los mercados para poder garantizar la diversificación de los cultivos. El borrador propuesto por la OMC en febrero de 2003 proponía una completa liberalización del comercio en productos de importancia específica para la diversificación de los cultivos ilegales de drogas y aquellos que son dañinos para la salud humana ${ }^{18}$. Oxfam solicita a los negociadores de la UE que se aseguren de que se amplía para garantizar oportunidades de mercado disponibles a los agricultores de países tropicales de manera que puedan diversificar y salir de los mercados que no funcionan adecuadamente, como el del café. En su conjunto, estas medidas asegurarían que los productores de café tuvieran acceso a los mercados locales, nacionales e internacionales de forma que la obtención de medios de vida sostenibles sea un propósito realista.

\section{Competencia limitada}

Los países Centroamericanos exportadores de café expresaron recientemente su preocupación de que:

'En términos de distribución de ingresos en la industria cafetalera mundial, la parte recibida por los países productores ha menguado significativamente, mientras los beneficios del sector importador se han multiplicado, dado que los bajos precios pagados a los productores no se han reflejado en los que se le cargan al consumidor final'19.

Existe un consenso creciente según el cual un problema fundamental del comercio del café reside en la estructura del mercado. Los desequilibrios de poder están siendo cada vez más reconocidos como un factor importante en el comercio internacional de materias primas en general. Cada vez se reconoce más la importancia de los desequilibrios de poder en el comercio internacional de materias primas. La FAO (Organización de NNUU para la Alimentación y la Agricultura) afirmaba recientemente que, 'La participación de los productores en los precios [de las materias primas] es inusualmente baja y parece ser aún más baja en el caso de las materias primas que tienen sectores de procesamiento de alimentos muy concentrados. Parece que la estructura del mercado es un área importante que debe ser investigada'20.

\footnotetext{
18 Primer Borrador de Modalidades, op.cit.

19 Párrafo 3 de 'Centroamérica se moviliza para eliminar el café de baja calidad', op. cit.

20 Consulta de la FAO, 'Back to Office Report by World Bank Official', (De vuelta al Informe de Oficina de los Funcionarios del Banco Mundial) Marzo 2002.
} 
Desde el final de los 80, la liberalización del mercado del café ha ido de la mano de un rápido incremento en la concentración empresarial. En 1998, las cuotas de mercado de las cuatro principales empresas tostadoras y de café instantáneo eran las siguiente: 25 por ciento de Philip Morris (recientemente ha cambiado su no mbre por Altria; el dueño de Kraft J acobs Suchard), 24 por ciento de Nestlé; y 7 por ciento para Sara Lee y para Procter \& Gamble. Es decir, entre las cuatro empresas acumulan el 63 por ciento del mercado mundial de café 21 . En cuanto a las compras de café verde, algunos intermediarios afirman que entre las cuatro empresas acumulan el 40 por ciento del volumen anual, encabezadas por Nestlé y Altria con un 13 por ciento cada una 22 . Resulta difícil explicar las diferencias entre esas cifras, excepto, quizás, por el mayor margen de las principales marcas, especialmente en el caso de los cafés solubles, como el Maxwell House de Altria/Kraft, Birds y Carte Noire, y el Nescafé de Nestlé.

La teoría económica ortodoxa considera que existe oligopolio cuando las cuatro empresas más grandes en un mercado tienen una cuota de mercado conjunta del 40 por ciento o superior ${ }^{23}$. Por consiguiente, parece que existe un oligopolio mundial en los mercados de café verde y consumidor. De acuerdo con la misma definición, el oligopolio también prevalece en el ámbito de la UE y dentro de muchos de los 15 Estados Miembros, donde la cuota de mercado combinada de las principales cinco compañías va desde el 72 por ciento en Austria hasta el 97 por ciento en Finlandia (el país con mayor consumo per cápita del mundo) ${ }^{24}$. De todas formas, difiere en forma llamativa entre países. Así, en el Reino Unido, con la preferencia de los consumidores por el café soluble, Nestlé tiene el 51 por ciento del mercado, mientras que en Holanda la subsidiaria de Sara Lee, Douwe Egberts, tiene el 69 por ciento, y en Suecia Altria/Kraft disfruta de un 53 por ciento de cuota de mercado 25 . Kraft es la que tiene la mayor cuota de mercado de todas, aproximadamente con un tercio del mercado total de la UE.

\footnotetext{
21 VAN DIJK ET AL., (1998).

22 Estas cifras provienen de diversas fuentes, entre ellas Volcafé.

23 El trabajo estándar es Scherer, (1980), que arguye (p. 67): ‘Cuando las cuatro empresas más grandes controlan el 40 por ciento o más del mercado total, es justo asumir que un oligopolio está comenzando a levantar la cabeza.'

24 International Trade Centre, (2002), p. 30.

25 Calfat y Flôres, (2002).
} 
Estas cifras explican bastante bien la menguante participación que se atribuye a los cultivadores de café y a los países productores en los precios mundiales. Enfrentados con parecidos limites a la competencia, los países industrializados han tomado medidas, desde finales del siglo XIX, para prevenir prácticas de oligopolio y, en algunos casos, para dividir las firmas implicadas. Hoy, a pesar de la economía global, no hay instituciones globales que lo hagan. Sin embargo, en ausencia de tal entidad, es mucho lo que la UE y sus Estados Miembros pueden hacer para combatir cualquier tendencia al oligopolio en el mercado europeo del café. La disminución del poder de las grandes empresas tostadoras permitiría al mercado respirar de nuevo y permitiría a los pequeños productores y trabajadores vivir dignamente gracias al ingreso de unos recursos mínimos procedentes del café que cultivan.

\section{Recomendaciones políticas}

La UE no debe permanecer pasiva mientras la crisis del mercado mundial del caféconducea un desastre del desarrollo mundial. La UE tienenumerosos instrumentos que pueden facilitar un cambio en las vidas de las personas pobres devastadas por esta crisis. Oxfam propone que la UE debería actuar en las siguientes áreas:

- Proporcionar 750 millones de euros en pagos desembolsables inmediatamente a todos los países ACP afectados por la crisis del café de acuerdo a sus necesidades.

- Aumentar los recursos de la ayuda a los cultivadores y trabajadores del café, utilizando recursos disponibles cuando sea posible. Esto ayudaría a los agricultores a la diversificación rural, la información de mercado, el desarrollo de cooperativas, los servicios de extensión, el acceso al crédito y a insumos agrícolas, y un plan para ayudarles a aprovechar al máximo los nichos del café de calidad.

- En el área del comercio internacional, la UE debe apoyar el Programa de Mejora de la Calidad de la OIC con el control independiente del café que entra en el mercado de la UE. Debe eliminar los aranceles existentes sobre el café procesado, así como todos los subsidios a la exportación y las cuotas en otros productos de los que los agricultores pobres pueden obtener ingresos para reemplazar los que han perdido por el café. Oxfam también solicita a los negociadores de la UE que se 
aseguren que dichas prácticas queden rápidamente prohibidas durante las actuales negociaciones de la Ronda de Doha.

- Apoyar la destrucción de algunos stocks de café de baja calidad para dar un inmediato y necesario empujón a los precios.

\section{Referencias bibliográficas}

Calfat, G. And R.G. FlôRes J R., (2002), “Government Actions to Support Coffee Producers - An Investigation of Possible Measures from the European Side", trabajo presentado en el Bitter Coffee Seminar (Seminario Café Amargo), Bruselas, Diciembre 2001, y publicado en Abril 2002.

FITTER, R. AND R. KAPLINSKY, (2001), "Who gains from product rents as the coffee market becomes more differentiated? A value chain analysis", IDS Bulletin 32(3): 69-82.

Food and Agriculture Organisation, (2002), "Back to office report by World Bank officials", Roma.

Oxfam, (2002), Mugged: Poverty in Your Coffee Cup, Oxford: Oxfam GB.

Scherer, F.M., (1980), Industrial Market Structure and Economic Performance (2nd edition), Boston (EEUU), Houghton Mifflin.

Sprey, L., (2002), "Effectiveness of Diversification for Smallholder Coffee Farmers", informe de investigación realizado para Oxfam Internacional en el Koninklijk Instituut voor de Tropen, Amsterdam.

Talbot, J.M., (1997), "Where does your coffee dollar go? The division of income and surplus along the coffee commodity chain", Studies in International Development 32(1): 56-91. 